http://dx.doi.org/10.32929/2446-8355.2019v28n1p111-122

\title{
INFLUÊNCIA DO TEOR DE ARGILA NAS RELAÇÕES ENTRE OS ATRIBUTOS QUÍMICOS DE SOLOS NO NOROESTE DO ESTADO DE SÃO PAULO
}

\author{
José Eduardo Soria ${ }^{1}$, Maria Julia Betiolo Troleis ${ }^{2}$, Marcelo Rodrigo Alves ${ }^{3}$, Rafael \\ Montanari $^{4 *}$, Marcelo Andreotti ${ }^{5}$
}

\author{
${ }^{1}$ Coordenador de Programas Integrados de Agricultura de Precisão, Empresa Timac Agro Paraguay, Cidade de \\ Hernandarias, PY \\ ${ }^{2}$ Doutoranda, Manejo e Conservação do Solo e da Água, Unesp, Ilha Solteira - SP \\ ${ }^{3}$ Docente, Qualidade do Solo, Unoeste - Presidente Prudente - SP \\ ${ }^{4}$ Professor Livre Docente, Manejo e Conservação do Solo e da Água, Unesp, Ilha Solteira - SP. * \\ r.montanari@unesp.br \\ ${ }^{5}$ Professor Livre Docente, Fertilidade do Solo, Unesp, Ilha Solteira - SP.
}

Recebido: 11/07/2018; Aceito: 11/04/2019

\begin{abstract}
RESUMO: O conhecimento das relações entre os atributos do solo e seu comportamento é imprescindível para compreensão de sua influência na produtividade das culturas. $\mathrm{O}$ estudo teve como objetivo avaliar a influência da granulometria em sua relação com atributos químicos na região noroeste do Estado de São Paulo. Foram avaliados 638 pontos amostrais, coletados em duas profundidades $(0-0,25$ e 0,25-0,50 m) compreendendo uma área de 18.600 ha, a qual foi avaliada por meio de análises de correlação e regressão para os atributos: teores de argila (AG), teor de matéria orgânica do solo (MO), capacidade de troca de cátions $(\mathrm{T}), \mathrm{pH}$ em água $(\mathrm{pH})$ e saturação de bases $(\mathrm{V}) . \mathrm{O}$ pH foi o atributo que apresentou a menor influência da variação da granulometria do solo. As relações, MOxT e $\mathrm{pHxV}$, apresentaram variações de comportamento com o aumento do teor de argila. A correlação entre atributos do solo e produtividade das culturas não pode ser observada isoladamente, visto que o comportamento destes atributos e suas relações são alterados com a mudança do teor de argila, proporcionando assim diferenças de relações solo-planta.
\end{abstract}

Palavras-chave: Correlação. Teor de matéria orgânica. Saturação por bases. pH. Capacidade de troca catiônica.

\section{INFLUENCE OF CLAY CONTENT IN RELATIONSHIP AMONG THE CHEMICAL SOIL PROPERTIES IN THE NORTHWEST OF THE STATE OF SÃO PAULO}

\begin{abstract}
Knowledge of relationship between soil attributes and their behavior is essential to understand their influence on crop productivity. The study aimed to evaluate the influence of soil texture in relation of its chemical attributes in the northwest of São Paulo State. Were evaluated 638 points collected in two depths $(0-0.25$ and $0,25-0,50 \mathrm{~m})$ comprising an area of 18,600 ha, which was assessed using correlation and regression analysis for attributes of clay content (AG), soil organic matter $(\mathrm{OM})$, cation exchange capacity $(\mathrm{T}) \mathrm{pH}$ in water $(\mathrm{pH})$ and bases saturation $(\mathrm{V})$. The $\mathrm{pH}$ was the attribute that had the lowest influence of soil texture variation. The relationships, MOxT and $\mathrm{pHxV}$, presented variations in behavior
\end{abstract}


with increasing clay content. The correlation between soil properties and crop yields cannot be seen in isolation, since the behavior these attributes and their relationships are modify with the change of clay content, providing differences in soil-plant relationships.

Key words: Correlation. Organic matter. Bases saturation. pH. Cations exchange capacity.

\section{INTRODUÇÃO}

O conhecimento sobre as propriedades químicas e físicas do solo e suas interações, associado a suas distribuições ao longo da paisagem, são de suma importância para o zoneamento agroecológico e consequentemente para a adoção de práticas adequadas de manejo, visando um melhor planejamento de uso da terra, maior eficiência na produção de alimentos e sustentabilidade do meio ambiente.

Corroborando com isso, Silva et al. (2010) afirmam que a interação entre atributos físicos e químicos do solo influenciam diretamente o crescimento e o desenvolvimento das culturas. Desta forma, a avaliação da variabilidade espacial destes atributos têm se tornado importante ferramenta na determinação de estratégias de manejo do solo, que procuram aumentar a produtividade agrícola (CARR, 2003; MINASNY; MCBRATNEY, 2007).

Os atributos do solo, químicos (acidez ou disponibilidade de nutrientes) ou físicos (granulometria, disponibilidade de água ou resistência à penetração das raízes), apresentam relações diretas ou inversas com a produtividade das culturas (SANA et al., 2014; CERRI; MAGALHÃES, 2012). Esses estudos, no entanto, apresentam variação em valores e ordem de importância nas relações solo-planta proporcionada, possivelmente, pela variação de solos, tipos de cultura ou manejo adotado.

Dalchiavon et al. (2011) verificaram que o aumento do teor de matéria orgânica e do pH do solo proporcionou aumento da produtividade de grãos de feijão em sistema de semeadura direta. Esses dois atributos do solo em particular apresentam grande correlação com outros atributos do solo que nem sempre obtêm valores de coeficientes de correlação significativos com a produtividade, a luz da estatística.

Em solos tropicais a matéria orgânica do solo é responsável por 75 a $90 \%$ da capacidade de troca de cátions do solo (BORTOLUZZI et al., 2009), portanto, o aumento de seu teor proporciona elevação na quantidade de cátions que o solo conseguirá reter (BRIEDIS et al., 2012). A capacidade de troca de cátions, juntamente com o pH, apresenta grande relação com o valor de saturação por bases do solo e sua disponibilidade de nutrientes às plantas (SANA et al., 2014). Sendo assim, qualquer outro atributo do solo que altere o comportamento destas relações, proporcionará mudanças na relação solo-planta.

O teor de argila está relacionado com vários outros atributos do solo (SORIA, 2014), influenciando suas dinâmicas e relações entre si ou solo-planta. Santos et al. (2011) observaram a influência da textura do solo na proteção física da matéria orgânica do solo e na formação de agregados. Cerri e Magalhães (2012) verificaram além das correlações do teor de argila com matéria orgânica do solo, também com a capacidade de troca de cátions, saturação por bases e a produtividade da cultura. Deste modo, a busca pelo entendimento destas 
relações entre os atributos do solo torna-se fundamental para se explicar variações encontradas nos estudos correlacionando o solo com a planta.

O presente trabalho teve como objetivo avaliar a influência do teor de argila do solo com atributos químicos na região noroeste do Estado de São Paulo, por meio de estudos de correlações lineares e regressões, para identificar as interações entre tais atributos e seu comportamento em diferentes classes de textura do solo.

\section{MATERIAL E MÉTODOS}

Para o presente trabalho, foram coletados 638 pontos amostrais em uma área de aproximadamente 18.600 hectares, cultivados com a cultura da cana-de-açúcar na região noroeste do estado de São Paulo, Brasil, entre as latitudes $20^{\circ} 20^{\prime} 20^{\prime \prime}$ e $20^{\circ} 45^{\prime} 51^{\prime \prime}$ Sul e as longitudes $51^{\circ} 01^{\prime} 10^{\prime \prime}$ e $51^{\circ} 31^{\prime} 30^{\prime \prime}$ Oeste.

Os pontos amostrais se encontram predominantemente sobre as formações do Grupo Bauru, que tem como principal rocha o arenito. Próximo às margens dos rios nas cotas mais baixas, são encontrados solos derivados de basalto, pertencente à formação Serra Geral (IPT, 1981). De acordo com a classificação de Köppen, o clima da região é definido como Aw, sendo caracterizado como tropical úmido com estação chuvosa no verão e seca no inverno, com temperatura variando entre 26,8 e $21,2^{\circ} \mathrm{C}$, precipitação pluvial média anual de $1.128 \mathrm{~mm}$ e chuvas concentradas no período de novembro a fevereiro (VIANELLO; ALVES, 2004).

A coleta das amostras de solos foi realizada no meio das entrelinhas da cultura, com o auxílio de um trado holandês, na densidade de um ponto amostral a cada 29 ha. Foram coletadas amostras deformadas em duas camadas (0-0,25 e 0,25-0,50 m, sendo denominadas respectivamente pelos algarismos arábicos 1 e 2, colocados logo após a sigla do atributo, para diferenciação das camadas analisadas). Essas amostras foram submetidas às determinações analíticas químicas e físicas em laboratório. Para análise do teor de argila (AG) utilizou-se o método da pipeta (CAMARGO et al., 1986). E para análise dos atributos químicos, foram utilizados os dados de matéria orgânica $(\mathrm{MO})$, capacidade de troca de cátions $(\mathrm{T}), \mathrm{pH}$ em água (pH) e saturação por bases (V), conforme Raij et al. (2001).

Para a análise dos dados, primeiramente, definiu-se os intervalos de classes de argila de 0 a 150,150 a 250,250 a 350 e maior que $350 \mathrm{~g} \mathrm{~kg}^{-1}$, os quais foram utilizados para o estudo de influência destas classes nos atributos químicos do solo. Realizou-se a análise descritiva para todos os dados e para cada classe de argila pré-definidas, onde foram calculados a média, mediana, moda, valores mínimos e máximos, desvio padrão e coeficiente de variação (SCHLOTZHAVER; LITTEL, 1997).

Foi gerado ainda uma matriz de correlação de Pearson, para as combinações, duas a duas, entre os atributos estudados, a qual foi realizada com os todos os atributos (AG, MO, T, pH e V) e para todos os pontos amostrais. Em seguida, avaliou-se a correlação linear entre os seguintes atributos químicos: matéria orgânica com capacidade de troca de cátions (MOxT); e, potencial hidrogeniônico com saturação por bases $(\mathrm{pHxV})$, análise esta realizada para as quatro classes de teor de argila. Também foram testados os modelos de regressão (linear, potencial, exponencial e quadrático) para as relações MOxT e pHxV, com todos os dados e 
para cada classe de argila avaliada, a fim de verificar a influência desta na relação entre os atributos químicos do solo. A escolha do melhor modelo de regressão foi realizada pelos valores mais elevados de $\mathrm{R}$ e $\mathrm{r}^{2}$. Os procedimentos estatísticos foram realizados pelo programa SAS - Statistical Analysis System (SCHLOTZHAVER; LITTEL, 1997).

\section{RESULTADOS E DISCUSSÃO}

Os atributos do solo apresentaram grande variabilidade entre os pontos amostrais, dentro da área de estudo, conforme verificado pelos valores máximos e mínimos na análise da estatística descritiva (Tabela 1). Os atributos físicos AG1 e AG2 apresentaram amplitudes de dados de 620 e $704 \mathrm{~g} \mathrm{~kg}^{-1}$, respectivamente, exibindo pontos amostrais classificados desde a classe arenosa até a muito argilosa.

Tabela 1. Análise descritiva dos atributos do solo analisados nas camadas de 0 a 0,25 e 0,25 a $0,50 \mathrm{~m}$ para os 638 pontos amostrais. Descriptive analysis of soil attributes analyzed in the 0 to 0,25 and 0,25 to 0,50 $\mathrm{m}$ layers for the 638 sampling points.

\begin{tabular}{|c|c|c|c|c|c|c|}
\hline \multirow{2}{*}{ Atributos } & \multicolumn{6}{|c|}{ Medidas estatísticas descritivas } \\
\hline & Média & Mediana & Máximo & Mínimo & $\mathrm{DP}^{(1)}$ & $\mathrm{CV} \%^{(2)}$ \\
\hline \multicolumn{7}{|c|}{ camada $0-0,25 \mathrm{~m}$} \\
\hline $\mathrm{AG} 1\left(\mathrm{~g} \mathrm{~kg}^{-1}\right)$ & 199 & 180 & 650 & 30 & 102 & 51,5 \\
\hline $\operatorname{MO1}\left(\mathrm{g} \mathrm{dm}^{-3}\right)$ & 20 & 18 & 81 & 5 & 8 & 41,7 \\
\hline pH1 & 5,6 & 5,6 & 7,7 & 3,7 & 0,8 & 13,7 \\
\hline $\mathrm{T} 1\left(\mathrm{mmol}_{\mathrm{c}} \mathrm{dm}^{-3}\right)$ & 62,6 & 53,8 & 287,2 & 31,1 & 30,5 & 48,8 \\
\hline $\mathrm{V} 1(\%)$ & 60 & 61 & 94 & 15 & 16 & 26,0 \\
\hline \multicolumn{7}{|c|}{ camada $0,25-0,50 \mathrm{~m}$} \\
\hline $\mathrm{AG} 2\left(\mathrm{~g} \mathrm{~kg}^{-1}\right)$ & 241 & 215 & 730 & 26 & 118 & 59,0 \\
\hline $\operatorname{MO} 2\left(\mathrm{~g} \mathrm{dm}^{-3}\right)$ & 13 & 12 & 40 & 4 & 5 & 37,2 \\
\hline $\mathrm{pH} 2$ & 5,5 & 5,5 & 7,2 & 3,7 & 0,7 & 13,6 \\
\hline $\mathrm{T} 2\left(\mathrm{mmol}_{\mathrm{c}} \mathrm{dm}^{-3}\right)$ & 52,3 & 46 & 190 & 20,1 & 24,2 & 46,3 \\
\hline $\mathrm{V} 2(\%)$ & 56 & 57 & 90 & 9 & 16 & 28,9 \\
\hline
\end{tabular}

(1) DP é o desvio padrão; ${ }^{(2)} \mathrm{CV} \%$ é o coeficiente de variação/ ${ }^{(1)} S D$ is the standard deviation; ${ }^{(2)} \mathrm{CV} \%$ is the coefficient of variation.

Para os atributos químicos T e MO foram verificados maiores valores médios e maior amplitude dos resultados para a camada 1 (0 a 0,25 m), mostrando o acúmulo de matéria orgânica na camada superficial e sua relação com a capacidade de troca de cátions em solos tropicais (BORTOLUZZI et al., 2009). Os valores de V\% apresentaram uma grande variação, ocorrendo na região desde solos com alto teor de nutrientes até solos pobres em nutrientes. Esta maior e menor fertilidade também pode ser verificada pelos valores de $\mathrm{pH}$. Sendo este último o atributo que apresentou o menor coeficiente de variação (CV\%). Campos et al. (2009) observaram variações semelhantes para os atributos AG, MO, T e V, em estudo realizado na mesma região do presente trabalho. Esta variação dos atributos analisados pode ser atribuída em parte (atributos químicos) ou totalmente (atributos físicos) aos processos de formação e gênese dos solos da área, com um destaque para à geologia da região (IPT, 1981), 
a qual originaram diferentes tipos de solos. Não pode ser descartado, para os atributos químicos, o efeito antrópico que é um fator de variação, com alteração principalmente nos atributos $\mathrm{pH}$ e V, durante o preparo das áreas para instalação e condução da cana-de-açúcar.

Quando analisada a estatística descritiva por classe de argila, nota-se uma tendência de aumento nas médias dos atributos químicos com o aumento do teor de argila (Tabelas 2 e 3). $\mathrm{O}$ pH é o único atributo que não apresentou elevação com o aumento do teor de argila, mantendo-se semelhante até seus valores de desvio padrão (DP) e CV\%. Para os demais atributos químicos, foi observada redução dos valores de DP e CV\%, na comparação com a Tabela 1. A redução nas medidas de dispersão dos dados quando agrupados nas classes de argila, pode ser devido a redução da variabilidade dos dados dentro de cada classe analisada.

Tabela 2. Análise descritiva dos atributos do solo analisados por classe de argila, na camada de 0 a 0,25 m. Descriptive analysis of soil attributes analyzed by clay class in the 0 to $0,25 \mathrm{~m}$ layer.

\begin{tabular}{|c|c|c|c|c|c|c|c|}
\hline \multirow{2}{*}{ Atributos } & \multicolumn{7}{|c|}{ Medidas estatísticas descritivas } \\
\hline & $\mathrm{n}^{(1)}$ & Média & Mediana & Máximo & Mínimo & DP & $\mathrm{CV} \%$ \\
\hline \multicolumn{8}{|c|}{ menor que $150 \mathrm{~g} \mathrm{~kg}^{-1}$} \\
\hline $\mathrm{AG} 1\left(\mathrm{~g} \mathrm{~kg}^{-1}\right)$ & 235 & 114,0 & 130,0 & 148,0 & 30,0 & 26,5 & 23,3 \\
\hline $\operatorname{MO1}\left(\mathrm{g} \mathrm{dm}^{-3}\right)$ & 235 & 16,9 & 15,7 & 40,0 & 5,0 & 6,0 & 35,5 \\
\hline $\mathrm{pH} 1$ & 235 & 5,6 & 5,6 & 7,7 & 3,7 & 0,8 & 14,3 \\
\hline $\mathrm{T} 1\left(\mathrm{mmol}_{\mathrm{c}} \mathrm{dm}^{-3}\right)$ & 235 & 50,1 & 46,6 & 172,3 & 29,3 & 15,6 & 31,2 \\
\hline V1 (\%) & 235 & 57,8 & 59,4 & 93,6 & 15,2 & 15,3 & 26,4 \\
\hline \multicolumn{8}{|c|}{$150-250 \mathrm{~g} \mathrm{~kg}^{-1}$} \\
\hline $\mathrm{AG} 1\left(\mathrm{~g} \mathrm{~kg}^{-1}\right)$ & 259 & 189,3 & 180,0 & 245,0 & 150,0 & 26,9 & 14,2 \\
\hline $\operatorname{MO1}\left(\mathrm{g} \mathrm{dm}^{-3}\right)$ & 259 & 18,9 & 17,7 & 45,5 & 6,0 & 6,3 & 33,5 \\
\hline $\mathrm{pH} 1$ & 259 & 5,5 & 5,5 & 7,6 & 4,0 & 0,8 & 14,2 \\
\hline $\mathrm{T} 1\left(\mathrm{mmol}_{\mathrm{c}} \mathrm{dm}^{-3}\right)$ & 259 & 56,7 & 51,4 & 251,8 & 28,4 & 22,2 & 39,1 \\
\hline V1 (\%) & 259 & 57,9 & 58,1 & 94,4 & 26,3 & 15,3 & 26,4 \\
\hline \multicolumn{8}{|c|}{$250-350 \mathrm{~g} \mathrm{~kg}^{-1}$} \\
\hline $\mathrm{AG} 1\left(\mathrm{~g} \mathrm{~kg}^{-1}\right)$ & 85 & 289,2 & 280,0 & 345,0 & 250,0 & 26,8 & 9,3 \\
\hline $\operatorname{MO} 1\left(\mathrm{~g} \mathrm{dm}^{-3}\right)$ & 85 & 22,8 & 21,1 & 50,0 & 10,0 & 7,8 & 34,3 \\
\hline $\mathrm{pH} 1$ & 85 & 5,6 & 5,7 & 7,4 & 3,8 & 0,7 & 12,5 \\
\hline $\mathrm{T} 1\left(\mathrm{mmol}_{\mathrm{c}} \mathrm{dm}^{-3}\right)$ & 85 & 76,5 & 70,0 & 179,3 & 38,0 & 25,8 & 33,8 \\
\hline $\mathrm{V} 1(\%)$ & 85 & 62,5 & 62,5 & 89,7 & 22,1 & 15,2 & 24,2 \\
\hline \multicolumn{8}{|c|}{ maior que $350 \mathrm{~g} \mathrm{~kg}^{-1}$} \\
\hline AG1 $\left(\mathrm{g} \mathrm{kg}^{-1}\right)$ & 59 & 444,5 & 430,0 & 650,0 & 350,0 & 68,9 & 15,5 \\
\hline $\operatorname{MO1}\left(\mathrm{g} \mathrm{dm}^{-3}\right)$ & 59 & 31,8 & 31,8 & 80,6 & 12,6 & 12,1 & 38,1 \\
\hline $\mathrm{pH} 1$ & 59 & 5,8 & 5,8 & 7,0 & 4,2 & 0,6 & 10,9 \\
\hline $\mathrm{T} 1\left(\mathrm{mmol}_{\mathrm{c}} \mathrm{dm}^{-3}\right)$ & 59 & 118,9 & 115,2 & 287,2 & 55,5 & 42,6 & 35,8 \\
\hline V1 (\%) & 59 & 72,9 & 74,8 & 93,8 & 31,2 & 12,0 & 16,4 \\
\hline
\end{tabular}


Este comportamento dos atributos químicos do solo analisados em relação às classes de argila pode ser explicado pelos coeficientes correlação $(\mathrm{R})$ apresentados na Matriz de correlação linear (Tabela 4). $\mathrm{O}$ atributo $\mathrm{pH} 2$ apresentou baixo valor de $\mathrm{R}$ e o $\mathrm{pH} 1$ não foi significativo com o teor de argila (AG), explicando a não variação dos dados médios por classe de argila (Tabelas 2 e 3), bem como a baixa influência deste atributo físico nos valores de $\mathrm{pH}$. Os atributos $\mathrm{MO}$ e $\mathrm{T}$ apresentaram as maiores correlações com a AG, para ambas as camadas, confirmando a grande interação do teor de argila na proteção da matéria orgânica do solo e a influência de ambas na capacidade do solo em reter cátions (SANTOS et al., 2011). Campos et al. (2010) analisando mapas de variabilidade de atributos do solo, verificaram que as regiões com os maiores teores de argila, apresentaram os maiores teores de $\mathrm{MO}$ e por sua vez, os maiores valores de T, confirmando a relação existente entre tais atributos.

Tabela 3. Análise descritiva dos atributos do solo analisados por classe de argila, na camada de 0,25 a 0,50 m. Descriptive analysis of the attributes of the soil analyzed by clay class, in the layer of 0.25 to $0.50 \mathrm{~m}$.

\begin{tabular}{|c|c|c|c|c|c|c|c|}
\hline \multirow{2}{*}{ Atributos } & \multicolumn{7}{|c|}{ Medidas estatísticas descritivas } \\
\hline & $\mathrm{n}^{(1)}$ & Média & Mediana & Máximo & Mínimo & DP & $\mathrm{CV} \%$ \\
\hline \multicolumn{8}{|c|}{ menor que $150 \mathrm{~g} \mathrm{~kg}^{-1}$} \\
\hline $\mathrm{AG} 2\left(\mathrm{~g} \mathrm{~kg}^{-1}\right)$ & 120 & 114,6 & 130,0 & 146,0 & 26,0 & 27,5 & 24,0 \\
\hline $\operatorname{MO} 2\left(\mathrm{~g} \mathrm{dm}^{-3}\right)$ & 120 & 12,2 & 11,3 & 26,0 & 4,0 & 4,5 & 37,1 \\
\hline $\mathrm{pH} 2$ & 120 & 5,5 & 5,6 & 7,2 & 4,0 & 0,7 & 13,4 \\
\hline $\mathrm{T} 2\left(\mathrm{mmol}_{\mathrm{c}} \mathrm{dm}^{-3}\right)$ & 120 & 40,6 & 38,5 & 68,3 & 20,1 & 10,0 & 24,6 \\
\hline $\mathrm{V} 2(\%)$ & 120 & 53,4 & 54,9 & 86,4 & 16,7 & 14,4 & 26,9 \\
\hline \multicolumn{8}{|c|}{$150-250 \mathrm{~g} \mathrm{~kg}^{-1}$} \\
\hline $\mathrm{AG} 2\left(\mathrm{~g} \mathrm{~kg}^{-1}\right)$ & 296 & 197,4 & 190,0 & 248,0 & 150,0 & 26,9 & 13,6 \\
\hline $\mathrm{MO} 2\left(\mathrm{~g} \mathrm{dm}^{-3}\right)$ & 296 & 12,3 & 11,1 & 33,0 & 4,0 & 4,1 & 33,5 \\
\hline $\mathrm{pH} 2$ & 296 & 5,4 & 5,4 & 7,2 & 3,7 & 0,8 & 14,2 \\
\hline $\mathrm{T} 2\left(\mathrm{mmol}_{\mathrm{c}} \mathrm{dm}^{-3}\right)$ & 296 & 45,1 & 42,7 & 121,7 & 25,4 & 13,3 & 29,5 \\
\hline $\mathrm{V} 2(\%)$ & 296 & 53,6 & 53,9 & 87,4 & 18,4 & 14,7 & 27,5 \\
\hline \multicolumn{8}{|c|}{$250-350 \mathrm{~g} \mathrm{~kg}^{-1}$} \\
\hline $\mathrm{AG} 2\left(\mathrm{~g} \mathrm{~kg}^{-1}\right)$ & 126 & 289,9 & 280,0 & 345,0 & 250,0 & 26,2 & 9,0 \\
\hline $\operatorname{MO} 2\left(\mathrm{~g} \mathrm{dm}^{-3}\right)$ & 126 & 13,7 & 13,0 & 29,0 & 5,3 & 4,2 & 30,5 \\
\hline $\mathrm{pH} 2$ & 126 & 5,4 & 5,4 & 7,1 & 3,7 & 0,7 & 13,7 \\
\hline $\mathrm{T} 2\left(\mathrm{mmol}_{\mathrm{c}} \mathrm{dm}^{-3}\right)$ & 126 & 54,2 & 49,4 & 142,6 & 28,7 & 19,5 & 35,9 \\
\hline $\mathrm{V} 2(\%)$ & 126 & 54,1 & 56,1 & 89,4 & 8,9 & 17,6 & 32,6 \\
\hline \multicolumn{8}{|c|}{ maior que $350 \mathrm{~g} \mathrm{~kg}^{-1}$} \\
\hline $\mathrm{AG} 2\left(\mathrm{~g} \mathrm{~kg}^{-1}\right)$ & 96 & 468,2 & 452,5 & 730,0 & 355,0 & 89,0 & 19,0 \\
\hline $\mathrm{MO} 2\left(\mathrm{~g} \mathrm{dm}^{-3}\right)$ & 96 & 18,0 & 17,0 & 39,8 & 7,0 & 6,3 & 34,9 \\
\hline $\mathrm{pH} 2$ & 96 & 5,8 & 5,9 & 7,2 & 4,0 & 0,6 & 9,9 \\
\hline $\mathrm{T} 2\left(\mathrm{mmol}_{\mathrm{c}} \mathrm{dm}^{-3}\right)$ & 96 & 86,5 & 78,4 & 190,0 & 36,2 & 35,1 & 40,5 \\
\hline $\mathrm{V} 2(\%)$ & 96 & 68,7 & 72,3 & 90,0 & 23,3 & 14,9 & 21,7 \\
\hline
\end{tabular}

(1) $\mathrm{n}$ representa o número de pontos amostrais $/^{(I)} n$ represents the number of sampling points. 
Tabela 4. Matriz de correlação linear simples entre os atributos do solo analisados nas camadas de 0 a 0,25 e 0,25 a 0,50 m. Simple linear correlation matrix between soil attributes analyzed in the 0 to 0.25 and 0,25 to 0.50 m layers.

\begin{tabular}{ccccc}
\hline \multicolumn{5}{c}{ camada de $0-0,25 \mathrm{~m}$} \\
\hline Atributos & $\mathrm{AG} 1$ & $\mathrm{MO} 1$ & $\mathrm{pH} 1$ & $\mathrm{~T} 1$ \\
\hline $\mathrm{MO} 1$ & $0,521^{* *}$ \\
$\mathrm{pH} 1$ & $0,043^{\mathrm{ns}}$ & $0,359^{* *}$ & & \\
$\mathrm{~T} 1$ & $0,648^{* *}$ & $0,732^{* *}$ & $0,316^{* *}$ & \\
$\mathrm{~V} 1$ & $0,261^{* *}$ & $0,436^{* *}$ & $0,792^{* *}$ & $0,549^{* *}$ \\
\hline \multicolumn{5}{c}{ camada de $0,25-0,50 \mathrm{~m}$} \\
\hline Atributos & $\mathrm{AG} 2$ & $\mathrm{MO} 2$ & $\mathrm{pH} 2$ & $\mathrm{~T} 2$ \\
\hline $\mathrm{MO} 2$ & $0,414^{* *}$ & & \\
$\mathrm{pH} 2$ & $0,133^{* *}$ & $0,325^{* *}$ & & \\
$\mathrm{~T} 2$ & $0,666^{* *}$ & $0,643^{* *}$ & $0,266^{* *}$ & \\
$\mathrm{~V} 2$ & $0,304 * *$ & $0,325^{* *}$ & $0,736^{* *}$ & $0,493^{* *}$ \\
\hline
\end{tabular}

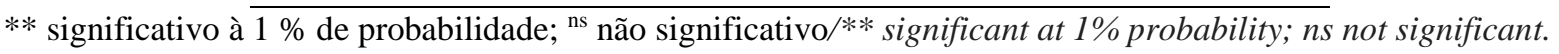

Ainda na Tabela 4, constata-se a alta correlação existente entre os atributos químicos: MO x T e pH x V. Esta interação é confirmada por diversos autores (CERRI; MAGALHÃES, 2012; SANA et al., 2014; SORIA, 2014), os quais demonstraram a importância da matéria orgânica do solo na criação de cargas negativas no solo e a relação existente entre a saturação de bases do solo e o seu $\mathrm{pH}$. Quando se faz uso destas relações entre os atributos químicos por classe de argila (Tabela 5), nota-se que a relação MOxT aumentou seu R com o aumento do teor de argila, condição não verificada para a relação $\mathrm{pHxV}$.

Tabela 5. Correlação linear simples entre os atributos do solo, para cada classe de argila nas camadas de 0 a 0,25 e 0,25 a 0,50 m. Simple linear correlation between soil attributes for each class of clay in the layers 0 to 0.25 and 0.25 to $0.50 \mathrm{~m}$.

\begin{tabular}{ccccc}
\hline \multirow{2}{*}{ Atributos } & $<150$ & $150-250$ & $250-350$ & $>350$ \\
\cline { 2 - 5 } & \multicolumn{5}{c}{ camada de $0-0,25 \mathrm{~m}$} \\
\hline MO1xT1 & $0,575^{* *}$ & $0,516^{* *}$ & $0,619^{* *}$ & $0,773^{* *}$ \\
$\mathrm{pH} 1 x \mathrm{x} 1$ & $0,784^{* *}$ & $0,836^{* *}$ & $0,769^{* *}$ & $0,780^{* *}$ \\
\hline \multicolumn{5}{c}{ camada de $0,25-0,50 \mathrm{~m}$} \\
\hline MO2xT2 & $0,539^{* *}$ & $0,522^{* *}$ & $0,569^{* *}$ & $0,640^{* *}$ \\
$\mathrm{pH} 2 x \mathrm{y} 2$ & $0,649^{* *}$ & $0,740^{* *}$ & $0,781^{* *}$ & $0,741^{* *}$ \\
\hline
\end{tabular}

** significativo à $1 \%$ de probabilidade/** significant at 1\% probability.

As regressões das relações entre atributos químicos MOxT e $\mathrm{pHxV}$ para os dados gerais são apresentados na Figura 1. Os modelos com melhor ajuste foram quadráticos (MO1xT1, pH1xV1 e pH2xV2) e exponencial (MO2xT2). Para as duas camadas, observa-se na relação MOxT, que o incremento do teor de matéria orgânica aumenta a capacidade do solo em reter cátions. $\mathrm{O}$ mesmo comportamento de relação direta foi observado na relação $\mathrm{pHxV}$, também para ambas as camadas, sendo verificado o aumento dos valores de $\mathrm{pH}$ juntamente com o 
aumento do valor de V\%. Também Cerri; Magalhães (2012) observaram as mesmas tendências diretas para estas relações.

Para as relações $\mathrm{pH} 1 \mathrm{xV} 1$ e $\mathrm{pH} 2 \mathrm{xV} 2$, ambas as parábolas apresentaram concavidade voltada para baixo, mostrando uma tendência de valor máximo para o atributo $\mathrm{V} \%$. As regressões de $\mathrm{pHxV}$ apresentaram menores erros padrão quando comparados com as regressões de MOxT.
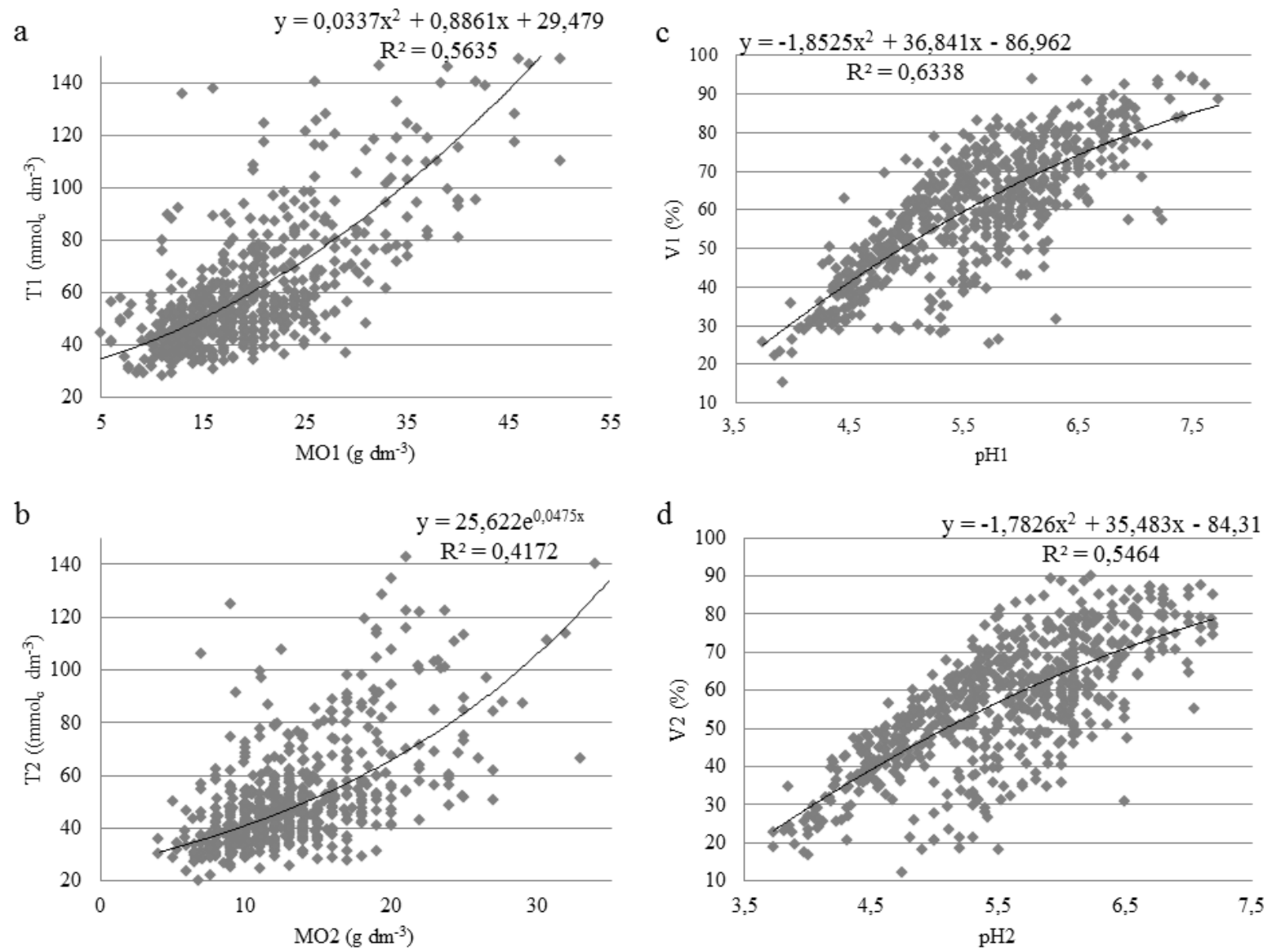

Figura 1. Modelos de regressões para as relações: a) relação MO1xT1; b) relação MO2xT2; c) relação $\mathrm{pH} 1 \times \mathrm{V} 1$; d) relação $\mathrm{pH} 2 \mathrm{xV} 2$. Regression models for the relations: a) MO1xT1 relation; b) MO2xT2 ratio; c) $p H 1 x V 1$ ratio; d) $p H 2 x V 2$ ratio.

Na geração dos modelos das relações MOxT e pHxV para as classes de argila, observase que os modelos que apresentaram os melhores ajustes, nem sempre são os mesmos quando se utiliza os dados em sua totalidade (Tabela 6). Apenas as relações MO2xT2 para a classe de argila 250-350 $\mathrm{g} \mathrm{kg}^{-1}, \mathrm{pH} 1 \mathrm{xV} 1$ para as classes de argila $250-350 \mathrm{~g} \mathrm{~kg}^{-1}$ e maior que $350 \mathrm{~g} \mathrm{~kg}^{-}$ , e pH2xV2 para a classe de argila maior que $350 \mathrm{~g} \mathrm{~kg}^{-1}$, exibiram os mesmos ajustes encontrados nas regressões geradas com todos os dados. Mostrando que as relações entre estes atributos químicos apresentaram de alguma forma uma influência da quantidade de argila presente no solo para a área estudada, alterando seu comportamento. 
Tabela 6. Modelos de regressão entre os atributos do solo analisados para cada classe de textura, nas camadas de 0 a 0,25 e 0,25 a 0,50 m. Regression models between the soil attributes analyzed for each texture class, in the layers 0 to 0.25 and 0.25 to $0.50 \mathrm{~m}$.

\begin{tabular}{|c|c|c|c|c|}
\hline & \multicolumn{4}{|c|}{ Modelo de regressão } \\
\hline & $\operatorname{Tipo}^{(1)}$ & Equação & $\mathrm{R}$ & $r^{2}$ \\
\hline Classe de textura & \multicolumn{4}{|c|}{ camada de $0-0,25 \mathrm{~m}$} \\
\hline \multicolumn{5}{|c|}{ MO1xT1 } \\
\hline menor que $150 \mathrm{~g} \mathrm{~kg}^{-1}$ & Exp. & $\mathrm{T} 1=31,038 \cdot \mathrm{e}^{0,0262 . \mathrm{MO} 1}$ & $0,599 * *$ & 0,359 \\
\hline $150-250 \mathrm{~g} \mathrm{~kg}^{-1}$ & Exp. & $\mathrm{T} 1=32,576 \cdot \mathrm{e}^{0,0268 . \mathrm{MO} 1}$ & $0,590 * *$ & 0,348 \\
\hline $250-350 \mathrm{~g} \mathrm{~kg}^{-1}$ & Exp. & $\mathrm{T} 1=42,221 \cdot \mathrm{e}^{0,024 \cdot \mathrm{MO} 1}$ & $0,620 * *$ & 0,385 \\
\hline maior que $350 \mathrm{~g} \mathrm{~kg}^{-1}$ & Lin. & $\mathrm{T} 1=32,5356+2,7243 . \mathrm{MO} 1$ & $0,773 * *$ & 0,597 \\
\hline \multicolumn{5}{|c|}{$\mathrm{pH} 1 \mathrm{xV} 1$} \\
\hline menor que $150 \mathrm{~g} \mathrm{~kg}^{-1}$ & Lin. & $\mathrm{V} 1=-25,88+14,93 \cdot \mathrm{pH} 1$ & $0,794 * *$ & 0,615 \\
\hline $150-250 \mathrm{~g} \mathrm{~kg}^{-1}$ & Lin. & $\mathrm{V} 1=-32,137+16,354 \cdot \mathrm{pH} 1$ & $0,836 * *$ & 0,700 \\
\hline $250-350 \mathrm{~g} \mathrm{~kg}^{-1}$ & Qua. & $\mathrm{V} 1=-165,11+66,035 \cdot \mathrm{pH} 1-4,46 \cdot \mathrm{pH} 1^{2}$ & $0,792 * *$ & 0,627 \\
\hline maior que $350 \mathrm{~g} \mathrm{~kg}^{-1}$ & Qua. & $\mathrm{V} 1=-208,75+83,382 \cdot \mathrm{pH} 1-4,9397 \cdot \mathrm{pH} 1^{2}$ & $0,819 * *$ & 0,671 \\
\hline \multicolumn{5}{|c|}{ camada de $0,25-0,50 \mathrm{~m}$} \\
\hline \multicolumn{5}{|c|}{$\mathrm{MO} 2 x \mathrm{~T} 2$} \\
\hline menor que $150 \mathrm{~g} \mathrm{~kg}^{-1}$ & Pot. & $\mathrm{T} 2=15,689 \cdot \mathrm{MO} 2^{0,3777}$ & $0,568 * *$ & 0,322 \\
\hline $150-250 \mathrm{~g} \mathrm{~kg}^{-1}$ & Lin. & $\mathrm{T} 2=24,333+1,6887 \cdot \mathrm{MO} 2$ & $0,522 * *$ & 0,273 \\
\hline $250-350 \mathrm{~g} \mathrm{~kg}^{-1}$ & Exp. & $\mathrm{T} 2=27,995 \cdot \mathrm{e}^{0,0443 \cdot \mathrm{MO} 2}$ & $0,583 * *$ & 0,339 \\
\hline maior que $350 \mathrm{~g} \mathrm{~kg}^{-1}$ & Lin. & $\mathrm{T} 2=22,326+3,5737 \cdot \mathrm{MO} 2$ & $0,640 * *$ & 0,409 \\
\hline \multicolumn{5}{|c|}{$\mathrm{pH} 2 \mathrm{xV} 2$} \\
\hline menor que $150 \mathrm{~g} \mathrm{~kg}^{-1}$ & Lin. & $\mathrm{V} 2=-15,959+12,527 \cdot \mathrm{pH} 2$ & $0,650 * *$ & 0,421 \\
\hline $150-250 \mathrm{~g} \mathrm{~kg}^{-1}$ & Lin. & $\mathrm{V} 2=-23,066+14,17 \cdot \mathrm{pH} 2$ & $0,740 * *$ & 0,548 \\
\hline $250-350 \mathrm{~g} \mathrm{~kg}^{-1}$ & Lin. & $\mathrm{V} 2=-46,696+18,604 . \mathrm{pH} 2$ & $0,781 * *$ & 0,610 \\
\hline maior que $350 \mathrm{~g} \mathrm{~kg}^{-1}$ & Qua. & $\mathrm{V} 2=-268,57+99,619 \cdot \mathrm{pH} 1-7,0998 \cdot \mathrm{pH} 2^{2}$ & $0,786^{* *}$ & 0,618 \\
\hline
\end{tabular}

(1) Tipos de modelos: Lin. - linear; Pot. - potencial; Exp. - exponencial; Qua. - quadrático; ** significativo à $1 \%$ de probabilidade/(1) Types of models: Lin. - linear; Pot. - potential; Exp. - exponential; Wed. - quadratic; ** significant at $1 \%$ probability.

Os coeficientes de determinação $\left(\mathrm{r}^{2}\right)$ aumentaram com o incremento do teor de argila para a relação MOxT, para as duas camadas. Este comportamento também foi verificado para o coeficiente R, apontando para uma maior influência de altos teores de argila na relação entre a matéria orgânica do solo e a capacidade de troca de cátions. Resultado este demonstrando a ação protetiva da MO pela argila e o efeito deste complexo coloidal no incremento da $\mathrm{T}$ do solo. Em contrapartida, este comportamento não é tão acentuado na relação $\mathrm{pHxV}$, sendo observado esta influência do teor de argila apenas na camada $2(0,25$ a $0,50 \mathrm{~m})$, a qual apresentou o mesmo aumento dos coeficientes $\mathrm{R}$ e $\mathrm{r}^{2}$, com o incremento do teor de argila. Esta variação entre as camadas para $\mathrm{pHxV}$ pode estar relacionada ao efeito antrópico, causado pelo manejo da fertilidade do solo que é mais acentuada na camada superficial do solo (0 a $0,25 \mathrm{~m}$ ), sendo que este manejo apresenta maior influência para os atributos $\mathrm{pH}$ e V. 
Na observação da plotagem dos modelos da relação entre os atributos químicos MOxT por classe de argila, ficou destacado o comportamento do aumento do teor de argila nesta relação (Figura 2a e 2b). Apesar de todos os modelos apresentarem relação direta entre MO e $\mathrm{T}$, suas linhas deslocam-se para cima com aumento do teor de argila. Ou seja, para um mesmo teor de matéria orgânica do solo, por exemplo, $24 \mathrm{~g} \mathrm{dm}^{-3}$, os solos da região apresentariam valores menores de capacidade de troca catiônica em solos de textura média e arenosos (60 $\left.\mathrm{mmol}_{\mathrm{c}} \mathrm{dm}^{-3}\right)$ e maiores valores deste atributo em solos de textura argilosa $\left(100 \mathrm{mmol}_{\mathrm{c}} \mathrm{dm}^{-3}\right)$. Isto pode ser devido à influência da argila nos atributos químicos MO e $\mathrm{T}$ verificados da Tabela 4, seja por causa da contribuição da atividade da argila (com presença de cargas negativas em sua superfície) e/ou a proteção que a argila confere a matéria orgânica do solo contra a mineralização, aumentando assim seu efeito em solos argilosos (SANTOS et al., 2011).
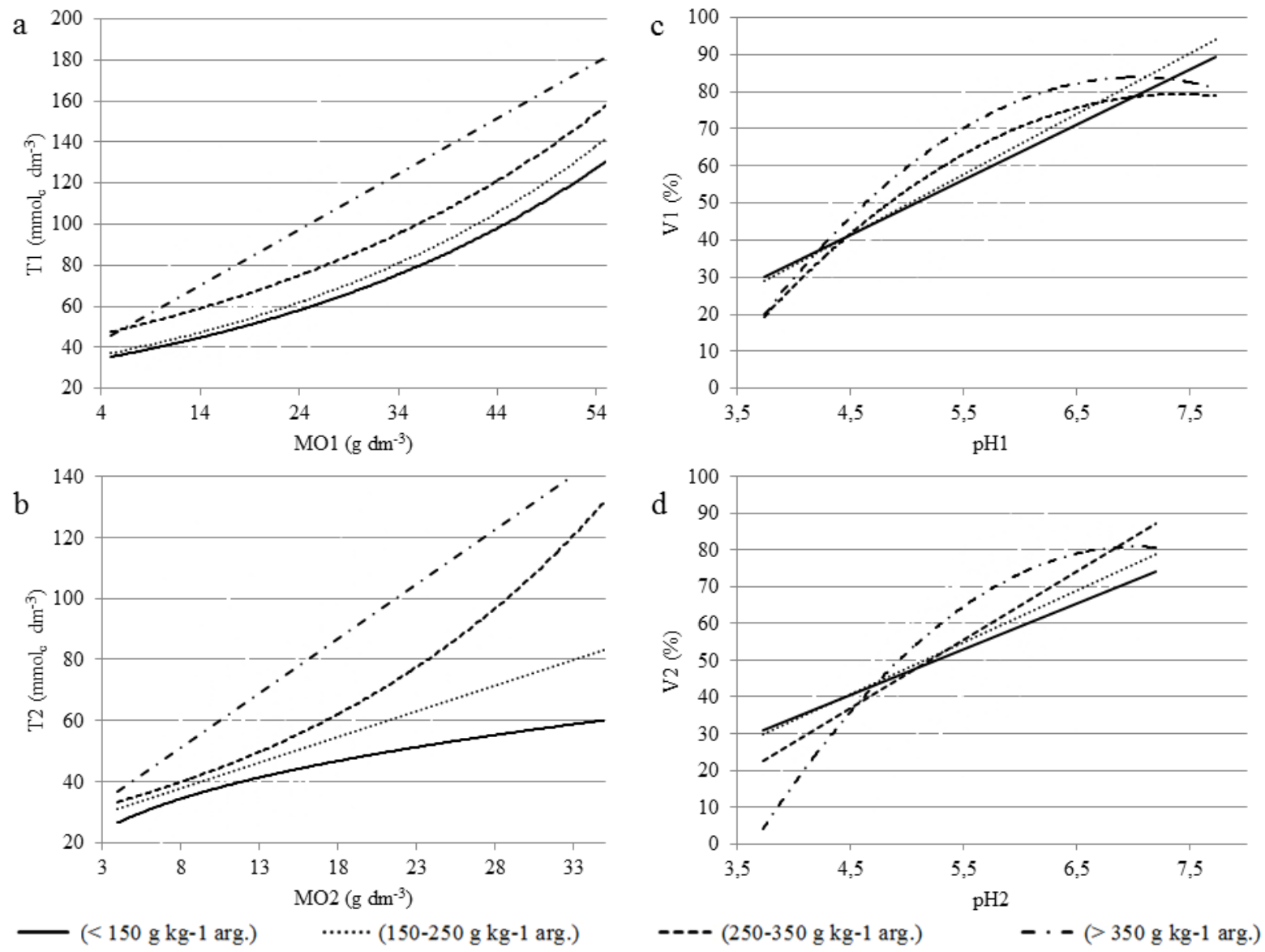

Figura 2. Modelos de regressões para as relações por classe de argila: a) relação MO1xT1; b) relação $\mathrm{MO} 2 x \mathrm{~T} 2$; c) relação $\mathrm{pH} 1 \mathrm{xV1}$; d) relação $\mathrm{pH} 2 \mathrm{xV} 2$. Regression models for relations by clay class: a) MO1xT1 relation; b) MO2xT2 ratio; c) pH1xV1 ratio; d) pH2xV2 ratio.

Os modelos da relação $\mathrm{pHxV}$ por classes de argila apresentaram comportamento diferente daquele visto para a relação MOxT. Para as classes de textura mais argilosa (250350 e maior que $350 \mathrm{~g} \mathrm{~kg}^{-1}$ ), nos valores mais baixos de $\mathrm{pH}$ (menores que 4,5), os modelos mostraram uma relação onde observa-se que, para um mesmo valor de $\mathrm{pH}$, solos com mais argila apresentam menores saturação por bases. Em contrapartida, no intervalo de pH entre 4,5 e 6,5 , onde se encontram aproximadamente $80 \%$ dos dados dos pontos amostrais, o 
comportamento inverte, ou seja, para um mesmo valor de $\mathrm{pH}$, os solos argilosos apresentam uma maior quantidade de bases presas nas cargas negativas dos colóides (maior saturação por bases) que os solos arenosos. Sendo assim, a definição de intervalos de $\mathrm{pH}$ que caracterizem sua disponibilidade de nutrientes, como a saturação por bases (V), deverá apresentar variação em relação ao tipo de solo e seu teor de argila.

$\mathrm{O}$ efeito do teor de argila na relação dos atributos $\mathrm{pHxV}$ foi menos pronunciado que na relação MOxT, isto pode ser explicado pelo maior coeficiente de correlação entre o teor de argila do solo com os atributos químicos $\mathrm{MO}$ e T, em comparação aos atributos pH e V.

\section{CONCLUSÃO}

O teor de argila do solo influencia de forma diferente as relações entre os atributos químicos MOxT e pHxV, sendo esta mais pronunciada na relação MOxT;

O aumento do teor de argila no solo proporciona uma maior inclinação na linha de tendência dos modelos matemáticos que expressam as regressões entre MOxT, conferindo um maior valor de $\mathrm{T}$ para o mesmo valor de $\mathrm{MO}$, quando se compara os solos arenosos $\mathrm{e}$ argilosos;

O comportamento da disponibilidade de nutrientes devido a variações do $\mathrm{pH}$, principalmente Cálcio e Magnésio (representado pela saturação por bases), apresenta variação em função do teor de argila do solo.

A correlação entre atributos do solo e a produtividade das culturas, não pode ser observada isoladamente, visto que o comportamento destes atributos e suas relações são alterados com a mudança do teor de argila.

\section{REFERÊNCIAS BIBLIOGRÁFICAS}

BORTOLUZZI, E. C.; RHEINHEIMER, D. S.; PETRY, C.; KAMINSKI, J. Contribuição de constituintes de solo à capacidade de troca de cátions obtida por diferentes métodos de extração. Revista Brasileira de Ciência do Solo, Viçosa, v. 33, n. 3, p.507-515, 2009.

BRIEDIS, C.; SÁ, J. C. M.; CAIRES, E. F.; NAVARRO, J. F.; INAGAKI, T. M.; FERREIRA, A. O. Carbono do solo e atributos de fertilidade em resposta à calagem superficial em plantio direto. Pesquisa Agropecuária Brasileira, Brasília, v. 47, n. 7, p.1007-1014, 2012.

CAMARGO, O. A.; MONIZ, A. C.; JORGE, J. A.; VAlADARES, J. M. A. S. Métodos de análise química, mineralógica e física de solos do Instituto Agronômico de Campinas. Campinas: Instituto Agronômico, 1986. 94 p.

CAMPOS, M. C. C.; MARQUES JUNIOR, J; PEREIRA, G. T. Influência das superfícies geomorfológicas na distribuição espacial dos atributos do solo em área sob cultivo de canade-açúcar. Pesquisa Agropecuária Tropical, Goiânia, v. 40, n. 2, p.133-141, 2010.

CAMPOS, M. C. C.; MARQUES JUNIOR, J; PEREIRA, G. T.; SOUZA, Z. M.; MONTANARI, R. Planejamento agrícola e implantação de sistema de cultivo de cana-de- 
açúcar com auxílio de técnicas geoestatísticas. Revista Brasileira de Engenharia Agrícola e Ambiental, Campina Grande, v. 13, n. 3, p.297-304, 2009.

CARR, J. R. Comments About Autokrigeability in Cokriging Based on an Empirical Study. Mathematical Geology, v. 35, n. 2, p.217-225, 2003.

CERRI, D. G. P.; MAGALHÃES, P. S. G. Correlation of physical and chemical attributes of soil with sugarcane yield. Pesquisa Agropecuária Brasileira, Brasília, v. 47, n. 4, p.613-620, 2012.

DALCHIAVON, F. C.; CARVALHO, M. C.; FREDDI, O. S.; ANDREOTTI, M.; MONTANARI, R. Variabilidade espacial da produtividade do feijoeiro correlacionada com atributos químicos de um Latossolo Vermelho Distroférrico sob sistema de semeadura direta. Bragantia, Campinas, v. 70, n. 4, p.908-916, 2011.

INSTITUTO DE PESQUISAS TECNOLÓGICAS DO ESTADO DE SÃO PAULO - IPT. Mapa geológico do Estado de São Paulo. v.1-2. São Paulo: Coordenadoria de Ensino Tecnológico - CET, 1981. 45 p.

MINASNY, B.; McBRATNEY, A. B. Spatial prediction of soil properties using EBLUP with the Matérn covariance function. Geoderma, v. 140, n. 4, p.324-336, 2007.

RAIJ, B. VAN.; ANDRADE, J. C.; CANTARELlA, H.; QUAGGIO, J. A. Análise química para avaliação da fertilidade de solos tropicais. Campinas: Instituto Agronômico, 2001. $285 \mathrm{p}$.

SANA, R. S.; ANCHINONI, I; BRANDÃO, Z. N.; HOLZSCHUH, M. J. Variabilidade espacial de atributos físico-químicos do solo e seus efeitos na produtividade do algodoeiro. Revista Brasileira de Engenharia Agrícola e Ambiental, Campina Grande, v. 18, n. 10, p.994-1002, 2014.

SANTOS, D. C.; PILlON, C. N.; FlORES, C. A.; LIMA, C. L. R.; CARDOSO, E. M. C.; PEREIRA, B. F.; MANGRICH, A. S. Agregação e frações físicas da matéria orgânica de um Argissolo Vermelho sob sistemas de uso no bioma Pampa. Revista Brasileira de Ciência do Solo, Viçosa, v. 35, n. 5, p.1735-1744, 2011.

SCHLOTZHAVER, S. D.; LITTELI, R. C. SAS: system for elementary statistical analysis. 2.ed. Cary: SAS, 1997. 441 p.

SILVA, S. A.; LIMA, J. S. S.; SOUZA, G. S.; OLIVEIRA, R. B.; SILVA, A. F. Variabilidade espacial do fósforo e das frações granulométricas de um Latossolo Vermelho Amarelo. Revista Ciência Agronômica, Fortaleza, v. 41, n. 1, p.1-8, 2010.

SORIA, J. E. Correlações lineares e espaciais entre atributos físico-químicos de solos de diferentes ambientes de produção de cana-de-açúcar no noroeste do estado de São Paulo. 2014. 97f. Dissertação (Mestrado em Agronomia) - Faculdade de Engenharia, Universidade Estadual Paulista, Ilha Solteira, 2014.

VIANELLO, R. L.; ALVES, A. R. Meteorologia básica e aplicações. Viçosa: UFG, 2004, $449 \mathrm{p}$. 